\title{
Poorly Differentiated Neuroblastoma
}

National Cancer Institute

\section{Source}

National Cancer Institute. Poorly Differentiated Neuroblastoma. NCI Thesaurus. Code C42047.

A neuroblastoma in which the differentiating neuroblasts constitute less than fivepercent of the tumor cells. 